\title{
Effects of CYP3A4 Inhibitors Ketoconazole and Verapamil and the CYP3A4 Inducer Rifampicin on the Pharmacokinetic Parameters of Fostamatinib: Results from In Vitro and Phase I Clinical Studies
}

\author{
Paul Martin ${ }^{6}$ Michael Gillen ${ }^{2} \cdot$ David Millson $^{1} \cdot$ Stuart Oliver $^{6}$ • \\ Clive Brealey ${ }^{1} \cdot$ Elliott B. Grossbard $^{3} \cdot$ Muhammad Baluom $^{3} \cdot$ David Lau $^{3}$. \\ David Sweeny ${ }^{3} \cdot$ Tim Mant $^{4} \cdot$ Kelli Craven ${ }^{5}$ \\ Published online: 6 January 2016 \\ (c) The Author(s) 2016. This article is published with open access at Springerlink.com
}

\begin{abstract}
Background Fostamatinib (R788) is a spleen tyrosine kinase (SYK) inhibitor. The active metabolite of fostamatinib, R406, is primarily metabolized by CYP3A4.

Objectives The aim of this study was to characterize hepatic microsomal metabolism of R406 and confirm the role of CYP3A4 in R406 metabolism, determining whether co-administration of CYP3A4 inhibitors (ketoconazole, verapamil) or inducers (rifampicin) affects R406 pharmacokinetics. Methods R406 stability was determined using human hepatic microsomes. The CYP450 isoforms responsible for R406 metabolism in humans were identified using expressed CYP450 isoforms and specific chemical inhibitors. The ketoconazole interaction study (double-blind, randomized, placebo-controlled, two-period crossover) involved fostamatinib administration (single 80-mg dose), alone and with ketoconazole (200 $\mathrm{mg}$ twice daily). The verapamil and rifampicin interaction studies (open-label, two-period, fixed-sequence) involved fostamatinib administration (single 150-mg dose), alone and with immediaterelease verapamil ( $80 \mathrm{mg}$ three times daily) or rifampicin
\end{abstract}

Michael Gillen

michael.gillen@astrazeneca.com

Paul Martin

paul.martin@astrazeneca.com

Former AstraZeneca, Macclesfield, UK

2 AstraZeneca, Gaithersburg, MD, USA

Rigel Pharmaceuticals, Inc, South San Francisco, CA, USA

4 Quintiles Drug Research Unit at Guy's Hospital, London, UK

5 Quintiles Phase I Unit, Overland Park, KS, USA

6 AstraZeneca Pharmaceuticals, Alderley Park, Macclesfield, Cheshire SK10 4TF, UK
(600 mg once daily). Standard pharmacokinetic parameters were calculated in all studies.

Results/Discussion Hepatic microsomes showed time-dependent loss of R406 and formation of para-O-demethylated R406. Microsomal metabolism of R406 was markedly inhibited by CYP3A4 inhibitors and, in the expressed CYP450 studies, the rate of R406 disappearance was greatest with CYP3A4. In the clinical studies, co-administration of ketoconazole caused a 2-fold (CI 1.77-2.30) increase in R406 exposure. Verapamil increased R406 exposure (39\% increase, CI 8-80), whereas rifampicin co-administration decreased exposure by $75 \%$ (CI 68-81). Fostamatinib was well tolerated. Conclusion The oxidative metabolism of R406 is predominantly catalyzed by CYP3A4. In clinical studies, exposure to R406 is affected by concomitant administration of CYP3A4 inducers/inhibitors. These findings should be taken into account when considering co-prescription of fostamatinib with such agents.

\section{Key Points}

The oxidative metabolism of R406 (the active metabolite of fostamatinib/R788) is predominantly catalyzed by CYP3A4.

Exposure to R406 is affected by concomitant administration of CYP3A4 inducers/inhibitors; coadministration of ketoconazole caused a 2-fold increase in R406 exposure, verapamil increased R406 exposure by $39 \%$, and rifampicin coadministration decreased exposure by $75 \%$.

The findings from these studies should be taken into account when considering co-prescription of fostamatinib with such agents. 


\section{Introduction}

Fostamatinib (previously known as R788) is an orally dosed spleen tyrosine kinase (SYK) inhibitor [1] that has completed phase III clinical trials as a therapy for the treatment of rheumatoid arthritis (RA) in patients who have shown inadequate response to traditional disease-modifying anti-rheumatic drugs or parenteral tumor necrosis factor- $\alpha$ antagonists [2-4].

Fostamatinib is a prodrug that is metabolized to its active metabolite, R406, by intestinal alkaline phosphatase [5]. R406 undergoes both direct glucuronidation and CYP3A4mediated para-O-demethylation to form the major metabolite, R529 [5]. R788 and R529 are much less active against syk than R406. Subsequent O-demethylations and dehydroxylation of R529 by gut bacteria lead to formation of the major excretory metabolite of R406, 3,5-benzene diol [5]. Activity of CYP3A4 is therefore integral to the metabolism of fostamatinib. Drugs can alter the activity of CYP3A4, acting either as inhibitors (e.g., ketoconazole [potent inhibitor], verapamil [moderate inhibitor]) or inducers (e.g. rifampicin [potent inducer]). These drugs may therefore alter the pharmacokinetics of any co-administered drug that is metabolized by this enzyme. Given the increased risk of co-morbidities for patients with RA, polypharmacy is commonly required [6-9]. The variety of concomitant medications may often include inhibitors or inducers of CYP3A4.

We report here the results of a series of in vitro studies designed to characterize the hepatic microsomal metabolism of R406 and to confirm the role of CYP3A4 in the metabolism of fostamatinib. We also performed clinical studies in which the CYP3A4 inhibitors ketoconazole (a potent inhibitor) and verapamil (a moderate inhibitor) and the CYP3A4 inducer rifampicin were co-administered with fostamatinib to healthy subjects to assess the potential for pharmacokinetic interactions. This was also intended to determine if any changes in the fostamatinib dose regimen would be needed if fostamatinib was co-administered with any of these three compounds in clinical practice. Ketoconazole, verapamil, and rifampicin are regarded as prototypical CYP3A4 modulators and are typically used in drug interaction studies that aim to determine the effect of CYP3A4 modulation on drug pharmacokinetics [10].

\section{Methods}

\subsection{In Vitro Experiments}

\subsubsection{Materials}

Human hepatic microsomes were obtained from Xenotech (Lenexa, KS, USA) and expressed CYP1A2, CYP2C9*1
+OR, CYP2C19 +OR, CYP2E1+OR+ cytochrome b5, CYP2D6*1+OR, and CYP3A4 +OR were purchased from Gentest (Woburn, MA, USA). The designation ' $+\mathrm{OR}$ ' signifies that the preparation contained supplemental, cDNA-expressed cytochrome P450 reductase. Ketoconazole, dextromethorphan, dextrorphan, diclofenac, phenacetin, acetamidophenol, testosterone, 6- $\beta$-hydroxytestosterone, midazolam, quinidine, sulfaphenazole, and nicotinamide adenine dinucleotide phosphate (NADPH) were acquired from Sigma Chemical Co (St Louis, MO, USA). S-(+)-mephenytoin, 4'-hydroxy-mephenytoin, 1'hydroxy-midazolam, and $4^{\prime}$-hydroxy-diclofenac were obtained from Ultrafine Chemicals (Manchester, UK). 3- $\mathrm{N}$ benzylnirvanol was purchased from Gentest. All other chemicals were the highest grades available.

\subsubsection{Microsomal Stability and Metabolite Profile of R406}

The stability of R406 was determined using human hepatic microsomes. Reactions were performed at $37{ }^{\circ} \mathrm{C}$ at a final volume of $502 \mu \mathrm{L}$ (445 $\mu \mathrm{L} \quad 0.1 \mathrm{M}$ phosphate buffer [pH 7.4], $5 \mu \mathrm{L}$ microsomes [0.1 mg protein], $2 \mu \mathrm{L} \mathrm{R} 406$ in dimethyl sulfoxide [DMSO; final concentration, $1 \mu \mathrm{M}$ ], and $50 \mu \mathrm{L}$ NADPH [final concentration, $1 \mathrm{mM}$ ]). Metabolic reactions were initiated by addition of the NADPH solution and stopped at $0,15,30$, and $50 \mathrm{~min}$. R406 concentrations were determined using high-performance liquid chromatography, followed by mass spectrometry in the multiple reaction monitoring (MRM) mode.

Metabolites were determined following incubation of $10 \mu \mathrm{M}$ R406 with human hepatic microsomes (1 mg protein/mL). Reactions were terminated after $15 \mathrm{~min}$. The precipitate was removed by centrifugation and $10 \mu \mathrm{L}$ of the supernatant was analyzed by liquid chromatography/mass spectrometry with ultraviolet $(254 \mathrm{~nm})$ detection.

\subsubsection{Metabolic Phenotyping}

Studies using expressed CYP450 isoforms and specific chemical inhibitors were performed to identify the CYP450 isoforms responsible for metabolism of R406 in humans. The chemical CYP450 inhibitors were ketoconazole (1 $\mu \mathrm{M}$, CYP3A4), troleandomycin $(100 \mu \mathrm{M}, \mathrm{CYP} 3 \mathrm{~A} 4)$, furafylline $(10 \mu \mathrm{M}, \quad$ CYP1A2), quinidine $(10 \mu \mathrm{M}$, CYP2D6), sulfaphenazole (10 $\mu \mathrm{M}$, CYP2C9), and 3- $N$ benzylnirvanol $(5 \mu \mathrm{M}, \mathrm{CYP} 2 \mathrm{C} 19)$. Incubation conditions for these microsomal metabolism studies were similar to those described above. All inhibitors were added in $1 \mu \mathrm{L}$ of methanol. Because of the time-dependent inactivation of CYP450 produced by furafylline and troleandomycin, these inhibitors were pre-incubated with the microsomes and NADPH for 15 min prior to addition of R406. Reactions were terminated at $0,15,30$, and $50 \mathrm{~min}$ and $\mathrm{R} 406$ 
concentrations were determined by mass spectrometry in MRM mode. First-order rate constants for R406 disappearance were calculated by linear regression using Microsoft Excel (Microsoft ${ }^{\circledR}$ Corporation, Redmond, WA, USA) and comparisons were made using these values.

For the expressed CYP450 studies, 20 pmol of CYP3A4, CYP1A2, CYP2D6*1, CYP2C19, CYP2C9*1, or CYP2E1 were added in place of the hepatic microsomes. All phenotyping reactions were terminated at $0,15,30$, and 50 min and analyzed by mass spectrometry in MRM mode. Rate calculations were based on the amount of R406 lost at the 15 -min time point.

\subsection{Clinical Studies}

\subsubsection{Subject Populations}

Healthy male subjects aged 18-45 years with a body mass index (BMI) between 19 and $28 \mathrm{~kg} / \mathrm{m}^{2}$ were eligible for enrollment in the ketoconazole study (C-935788-001). In the rifampicin (NCT01336218) and verapamil (NCT01197781) studies, eligibility criteria were similar: healthy male or female subjects aged $18-55$ years with a minimum weight of $50 \mathrm{~kg}$ and BMI between 18 and $35 \mathrm{~kg} /$ $\mathrm{m}^{2}$.

Exclusion criteria for the three studies included a history or the presence of any clinically relevant condition, including gastrointestinal, renal, or hepatic disease; recent administration of drugs with enzyme-inducing properties (e.g., St John's Wort); a history of excessive alcohol consumption; use of any investigational drug and/or participation in any clinical trial within 4 months (ketoconazole study) or 1 month (verapamil and rifampicin studies) of first dosing; and previous administration of fostamatinib (R788). In the verapamil and rifampicin studies, subjects were also excluded if they had an absolute neutrophil count of $<2500 / \mathrm{mm}^{3}$ at screening.

\subsubsection{Clinical Study Designs}

The designs of the three clinical studies, each of which was conducted at a single center, are summarized in Table 1.

The ketoconazole interaction study was a double-blind, randomized, placebo-controlled, two-period crossover trial in which eight male subjects received a single dose of fostamatinib $(80 \mathrm{mg})$ on the second of 4 days of treatment with either ketoconazole (200 mg twice daily) or placebo. In this study, fostamatinib was administered as a suspension in orange juice. The verapamil and rifampicin interaction studies were both open-label, two-period, fixedsequence trials that enrolled 15 male or female subjects. In period 1 of each study, the only drug administered was a single 150-mg dose of fostamatinib (administered on day 1 of period 1). In period 2 of each study, a single $150-\mathrm{mg}$ dose of fostamatinib was administered in conjunction with immediate-release verapamil $(80 \mathrm{mg}$ three times daily from day 1 to day 4 of period 2, with fostamatinib administered on day 2) or rifampicin (600 $\mathrm{mg}$ once daily from day 1 to day 8 of period 2, with fostamatinib administered on day 6). In these studies, fostamatinib was administered in tablet form. Suitable washout periods were employed between sequential doses of fostamatinib in all studies (Fig. 1). In phase III trials, fostamatinib doses of $150 \mathrm{mg}$ once daily and $100 \mathrm{mg}$ twice daily were used and therefore a dose of $150 \mathrm{mg}$ was chosen to assess drug interactions in the current studies with rifampicin and verapamil which were run relatively late in development. A dose of fostamatinib $80 \mathrm{mg}$ was used in the ketoconazole study as this study was run earlier in development before a phase II/III dose was set.

\subsubsection{Study Endpoints}

The objective of the ketoconazole study was to assess the effects of multiple oral doses of ketoconazole on the singledose pharmacokinetics of fostamatinib (R788) and its metabolite, R406. The primary aims of the verapamil and rifampicin interaction studies were to assess the pharmacokinetics of R406 in healthy subjects when fostamatinib was administered alone and in combination with verapamil or rifampicin. All three studies also examined the safety and tolerability of fostamatinib when given in combination with ketoconazole, verapamil, or rifampicin.

\subsubsection{Pharmacokinetic Assessments}

Whole blood was collected via venous catheter into ethylene diamine tetra acetate. Samples were chilled prior to centrifugation (speed, $1500 \mathrm{~g}$; temperature, $4{ }^{\circ} \mathrm{C}$; duration, $10 \mathrm{~min}$; performed within $30 \mathrm{~min}$ of sampling) and plasma was collected and stored at $-80{ }^{\circ} \mathrm{C}$ within $30 \mathrm{~min}$ of centrifugation. In the ketoconazole interaction study, blood samples were collected for pharmacokinetic analysis prior to, and between 0.25 and $120 \mathrm{~h}$ after, fostamatinib administration (Table 1). A pre-fostamatinib-dose blood sample was also collected in the verapamil and rifampicin interaction studies and the period over which blood samples were collected was extended from 0.5 to $96 \mathrm{~h}$ postdose in these studies (Table 1).

Validated liquid chromatography tandem mass spectrometry methods were used to determine the plasma levels of R406 in all three clinical studies, and of R788 in the ketoconazole interaction study. In the verapamil and rifampicin interaction studies, the lower limit of quantitation (LLOQ) for R406 was $2.50 \mathrm{ng} / \mathrm{mL}$. In the 
Table 1 Schedule of drug administration and pharmacokinetic assessments in the three clinical studies

\begin{tabular}{lllllllllll}
\hline Study & \multicolumn{1}{l}{ Day } & & & & & & & & \\
\cline { 2 - 7 } & 1 & 2 & 3 & 4 & 5 & 6 & 7 & 8 & 9 & 10
\end{tabular}

Ketoconazole interaction study

Periods 1 and 2

Drug administration

Ketoconazole (200 mg twice daily) or placebo ${ }^{\mathrm{a}}++_{+}+$

Fostamatinib $(80 \mathrm{mg})^{\mathrm{b}}$

Pharmacokinetic assessments ${ }^{\mathrm{c}}$

Verapamil interaction study

Period 1

Drug administration

Fostamatinib $(150 \mathrm{mg})^{\mathrm{d}}$

Pharmacokinetic assessments ${ }^{\mathrm{e}}$

Period 2

Drug administration

IR verapamil ( $80 \mathrm{mg}$ three times daily)

Fostamatinib $(150 \mathrm{mg})^{\mathrm{d}}$

Pharmacokinetic assessments ${ }^{\mathrm{e}}$

Rifampicin interaction study

Period 1

Drug administration

Fostamatinib $(150 \mathrm{mg})^{\mathrm{d}}$

Pharmacokinetic assessments ${ }^{\mathrm{e}}$

Period 2

Drug administration

Rifampicin (600 mg once daily)

Fostamatinib $(150 \mathrm{mg})^{\mathrm{d}}$

Pharmacokinetic assessments ${ }^{\mathrm{e}}$

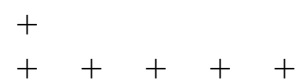

$+\quad++$

$+$

$+++++$

$+$

$+\quad+\quad++$

$+\quad+\quad+\quad+\quad+\quad+$

$+$

$+\quad+\quad+$

$I R$ immediate release

${ }^{a}$ First dose administered on evening of day 1, last dose on evening of day 4

b Single dose administered as powder in orange juice 120 min after completion of breakfast and $135 \mathrm{~min}$ after administration of ketoconazole/placebo

${ }^{c}$ Blood samples collected prior to and $0.25,0.5,1,1.5,2,4,6,8,12,16,24,32,48,56,72,96$, and $120 \mathrm{~h}$ after fostamatinib administration

d Single dose $(3 \times 50$-mg tablets $)$ administered with water; administered at the same time as verapamil/ rifampicin in period 2; all subjects required to fast from $10 \mathrm{~h}$ prior to until $4 \mathrm{~h}$ after administration of fostamatinib

e Blood samples collected prior to and $0.5,1,1.5,2,3,4,6,8,12,16,24,36,48,72$, and $96 \mathrm{~h}$ after fostamatinib administration ketoconazole interaction study, the LLOQs for R788 and R406 were 0.5 and $0.25 \mathrm{ng} / \mathrm{mL}$, respectively.

For the verapamil study, the precision $(\% \mathrm{CV})$ and accuracy ( $\%$ bias) for the quality control samples at three concentrations were $\leq 3.5 \%$ and were within -7.5 to $0.9 \%$. For the rifampicin study, the precision and accuracy were $\leq 6.9 \%$ and were within -6.5 to $-4.0 \%$, respectively.

For the ketoconazole study, the precision and accuracy were $\leq 13.7 \%$ and were within -10.9 to $-1.0 \%$, respectively.

\subsubsection{Pharmacokinetic Analysis}

Pharmacokinetic parameters were derived using standard non-compartmental methods. In the ketoconazole interaction study, all samples were assayed for R406 levels but, because previous work had shown that plasma R788 concentrations are negligible after oral fostamatinib administration, only selected samples were assayed for R788. In all studies, the maximum plasma concentration $\left(C_{\max }\right)$, time to $C_{\max }\left(t_{\max }\right)$, terminal half-life $\left(t_{1 / 2}\right)$, and area under the curve from zero to infinity $\left(\mathrm{AUC}_{0-\infty}\right)$ for R406 were determined. 

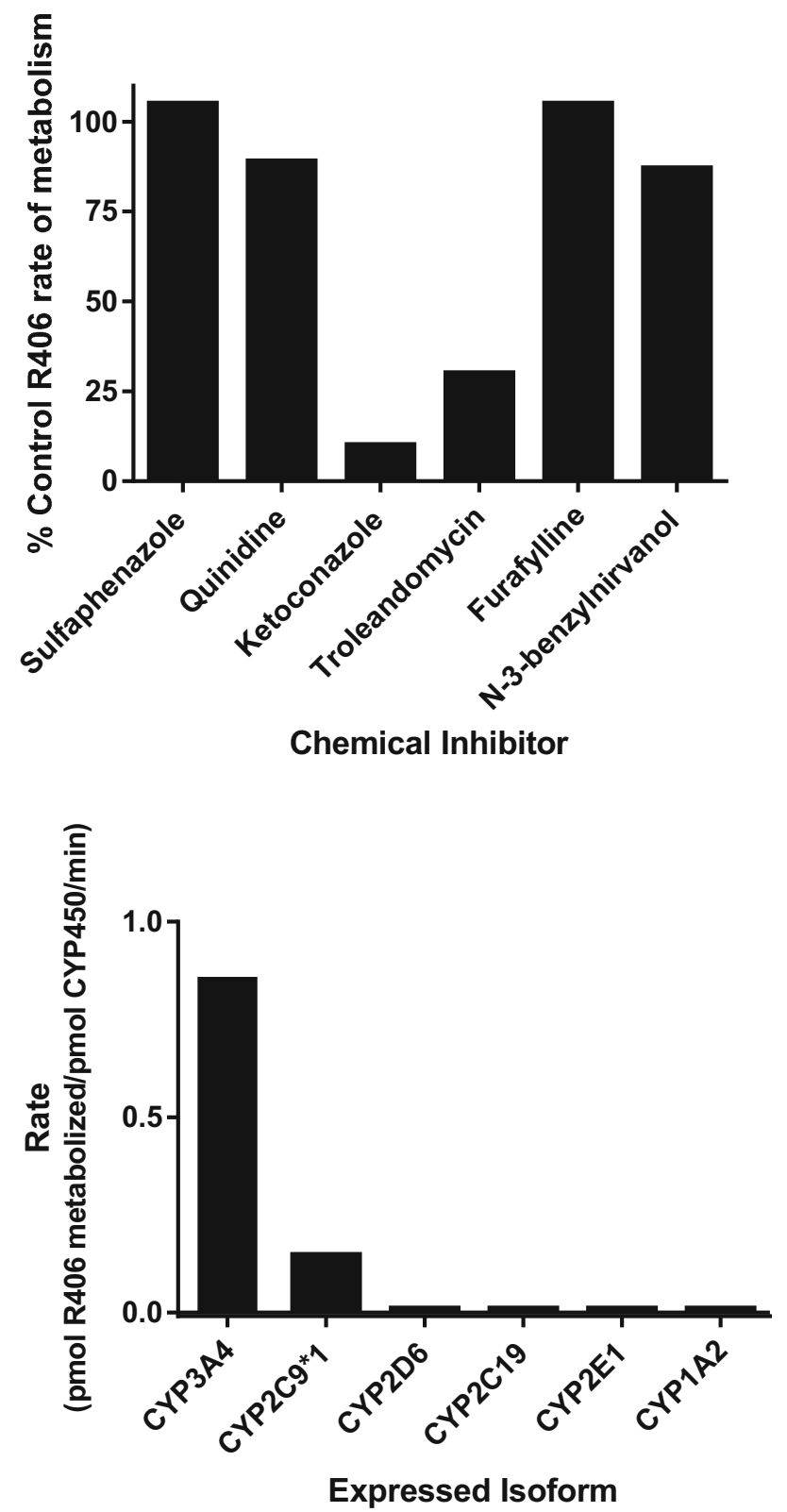

Fig. 1 Determination of the CYP450 isoforms involved in R406 metabolism by using specific chemical inhibitors and expressed enzymes

In the verapamil and rifampicin interaction studies, the pharmacokinetics of R406, but not R788, were determined.

\subsubsection{Safety Assessments}

In all three studies, safety and tolerability assessments included the incidence and severity of adverse events (AEs), vital signs, physical examination, clinical laboratory data, and electrocardiograms. AEs were tabulated and summarized according to the Medical Dictionary for Regulatory Activities (MedDRA).

\subsubsection{Statistical Analysis}

In the ketoconazole interaction study, the sample size of eight (four subjects randomized to each sequence in a twoway crossover design) was chosen to allow detection of clinically significant increases in exposure $(95 \%$ power to detect a $50 \%$ increase in exposure [one-sided test at $5 \%$ significance level], assuming a within-subject coefficient of variation $[\mathrm{CV}]$ of $25 \%$; $>99 \%$ power to detect doubling of exposure). The verapamil and rifampicin interaction studies were not powered to allow pursuit of a claim of no effect of verapamil/rifampicin on exposure to R406 (possible if the treatment ratio $90 \%$ confidence intervals [CIs] were between $80 \%$ and $125 \%$ ). Therefore, the results of the verapamil and rifampicin interaction studies were interpreted based on the size of the treatment ratio and associated $90 \% \mathrm{CI}$. Assuming inter-subject CVs of $27 \%$ for AUC and $29 \%$ for $C_{\max }$ (data based on a previous unpublished study), it was estimated that 12 subjects would provide $80 \%$ power to detect treatment ratios of $\geq 134 \%$ (verapamil) and $\leq 75 \%$ (rifampicin) in these two parameters (two-sided test at $5 \%$ significance level).

In the ketoconazole interaction study, all subjects who received at least one dose of fostamatinib were included in the pharmacokinetic analysis and all subjects who received treatment were included in the safety analysis. Pharmacokinetic data were summarized using descriptive statistics including $n$, mean, $\mathrm{SD}$, median, minimum ( $\mathrm{min}$ ), and maximum $(\max )$ values. Additional analyses (calculation of treatment ratios and CIs) were performed to investigate the effect of ketoconazole co-administration on the AUC and $C_{\max }$ of R406.

In the verapamil and rifampicin interaction studies, the pharmacokinetic analysis sets included only subjects who had received at least one dose of fostamatinib and who had at least one post-dose pharmacokinetic measurement without important protocol deviations or violations that might substantially affect pharmacokinetics (e.g., vomiting after drug administration). The safety sets in these studies comprised all subjects who had received at least one dose of fostamatinib and for whom at least one post-dose safety assessment was available. Quantitative continuous variables were summarized using descriptive statistics. Additionally, for all pharmacokinetic parameters except $t_{\max }$, geometric means and geometric CVs were reported and categorical variables were summarized in frequency tables.

The effects of verapamil and rifampicin on R406 were determined by analyzing $C_{\max }$ and AUC data from subjects with valid data from both periods 1 and 2 using an analysis of variance model with fixed effects for treatment and subject. Least-squares geometric means, two-sided $95 \%$ CIs, and ratios of geometric means, together with twosided $90 \%$ CIs of test treatment (fostamatinib plus 
verapamil/rifampicin) and reference treatment (fostamatinib alone) were estimated.

In the ketoconazole interaction study, pharmacokinetic parameters were analyzed using WinNonlin Professional (Pharsight Corporation, Cary, NC, USA; Version 4.1) and Microsoft ${ }^{\circledR}$ Excel (Microsoft ${ }^{\circledR}$ Corporation, Redmond, CA, USA). In the verapamil and rifampicin interaction studies, all analyses were conducted using SAS $^{\circledR}$ v9.2 (SAS Institute Inc, Cary, NC, USA), WinNonlin ${ }^{\circledR}$ Professional v5.2 (Pharsight Corp, Mountain View, CA, USA), and SigmaPlot ${ }^{\circledR}$ v9.01 (verapamil)/v9.0 (rifampicin) (Systat Software Inc, San Jose, CA, USA).

\section{Results}

\subsection{In Vitro Experiments}

\subsubsection{Microsomal Stability and Metabolite Profile of R406}

Hepatic microsomes showed time-dependent loss of R406 (microsomal half-life, $24.4 \mathrm{~min}$ ). Liquid chromatography/mass spectrometry showed that un-metabolized R406 eluted at $40 \mathrm{~min}$ and the major metabolite (para-Odemethylated R406, R529) eluted at $34 \mathrm{~min}$. Other unidentified metabolites were also observed.

\subsubsection{Metabolic Phenotyping}

The studies involving CYP450 inhibitors and expressed CYP450 isoforms demonstrated that CYP3A4 plays a major role in the oxidative metabolism of R406. Microsomal metabolism of R406 was markedly inhibited by ketoconazole and troleandomycin (CYP3A4 inhibitors) whereas all other inhibitors had a minimal effect on R406 disappearance (Fig. 1a). Moreover, in the expressed CYP450 studies, the rate of disappearance of R406 (pmol R406 metabolized/pmol CYP450/min) was greatest with CYP3A4 (Fig. 1b). In these studies, only expressed CYP3A4 and CYP2C9*1 were found to metabolize R406, but the rate of R406 disappearance was 5.6-fold greater with CYP3A4 than with CYP2C9*1.

\subsection{Clinical Study}

\subsubsection{Subject Demographics}

All subjects who enrolled in the ketoconazole and rifampicin interaction studies ( $N=8$ and $N=15$, respectively) completed the study per protocol. Three of the 15 subjects who enrolled in the verapamil interaction study withdrew and 12 completed. No withdrawals from the study were because of safety reasons. Baseline characteristics of all subjects are shown in Table 2. All 38 subjects were male.

\subsection{Pharmacokinetics}

\subsubsection{Ketoconazole Interaction Study}

No pharmacokinetic parameters are reported for the parent compound because plasma levels of R788 were negligible, both when fostamatinib was administered with ketoconazole-placebo and when it was administered in conjunction with ketoconazole. The highest concentration of R788 obtained in any subject was $<7 \mathrm{ng} / \mathrm{mL}$, and no quantifiable R788 (i.e., above $0.5 \mathrm{ng} / \mathrm{mL}$ ) was obtained beyond $2 \mathrm{~h}$
Table 2 Baseline demographic and subject characteristics

\begin{tabular}{llll}
\hline Characteristic & \multicolumn{2}{l}{ Interaction study } & \\
\cline { 2 - 4 } & $\begin{array}{l}\text { Ketoconazole } \\
N=8\end{array}$ & $\begin{array}{l}\text { Verapamil } \\
N=15\end{array}$ & $\begin{array}{l}\text { Rifampicin } \\
N=15\end{array}$ \\
\hline Gender & & & \\
Male, $n(\%)$ & $8(100)$ & $15(100)$ & $15(100)$ \\
Female, $n(\%)$ & $0(0.0)$ & $0(0.0)$ & $0(0.0)$ \\
Age, years; mean (SD) & $23(3)$ & $32(12)$ & $33(10)$ \\
Race & & & \\
White, $n$ (\%) & $8(100.0)$ & $7(46.7)$ & $8(53.3)$ \\
Black/African American, $n(\%)$ & $0(0.0)$ & $6(40.0)$ & $6(40.0)$ \\
American Indian/Alaskan Native, $n(\%)$ & $0(0.0)$ & $2(13.3)$ & $0(0.0)$ \\
Asian, $n$ (\%) & $0(0.0)$ & $0(0.0)$ & $1(6.7)$ \\
Height, m; mean (SD) & $1.82(0.05)$ & $1.77(0.07)$ & $1.76(0.04)$ \\
Weight, kg; mean (SD) & $78.0(6.9)$ & $86.6(15.3)$ & $80.3(10.4)$ \\
BMI, kg/m ${ }^{2}$; mean (SD) & $23.5(1.6)$ & $27.5(3.4)$ & $25.9(3.8)$ \\
\hline
\end{tabular}

$B M I$ body mass index, $S D$ standard deviation 

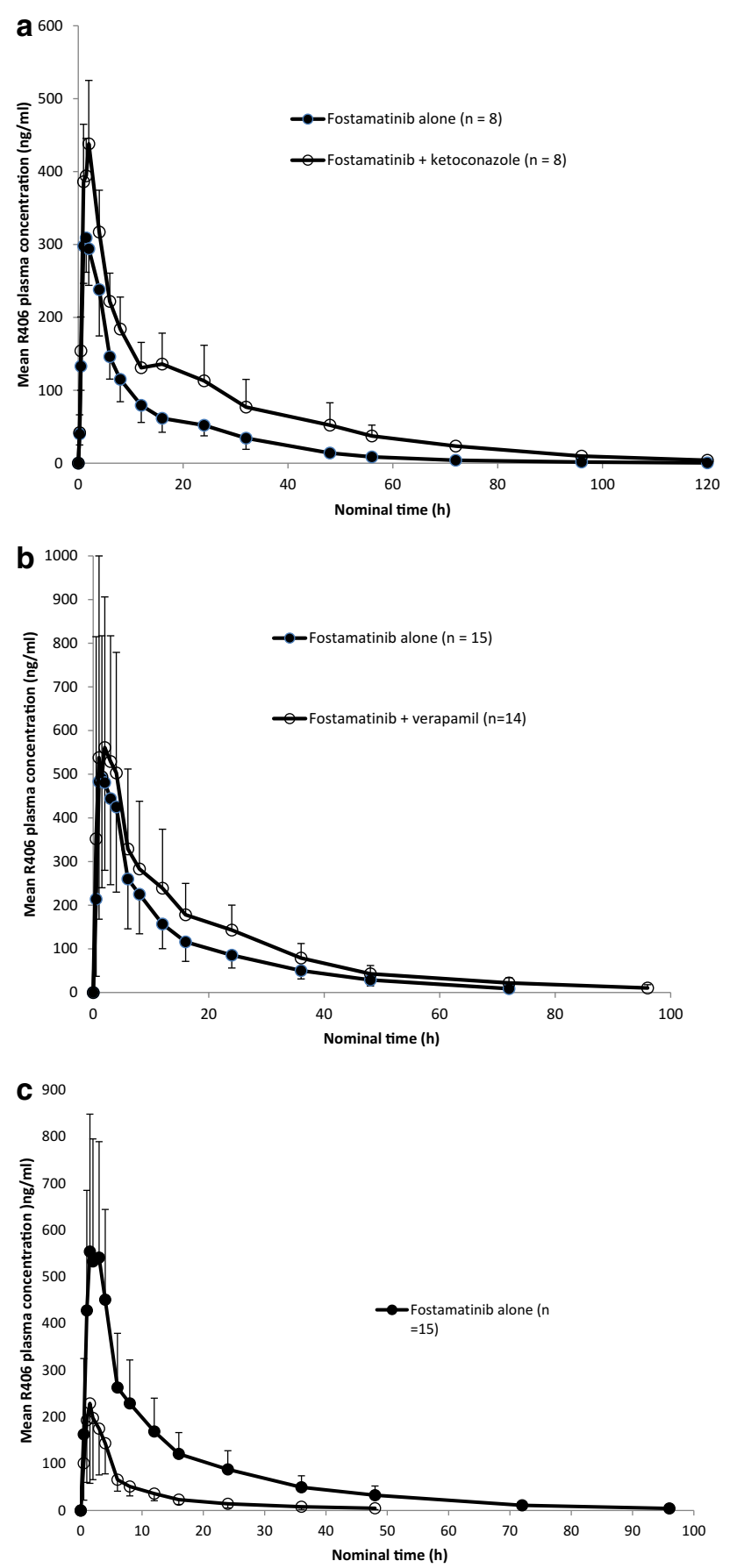

Fig. 2 Mean R406 concentration-time profiles in the three clinical studies. a Ketoconazole interaction study. b Verapamil interaction study. c Rifampicin interaction study

post-dose in any subject. Co-administration of ketoconazole (200 mg twice daily) increased plasma R406 exposure after administration of $80 \mathrm{mg}$ fostamatinib (Figs. 2a, 3a). Average increases in $C_{\max }$ and $\mathrm{AUC}_{0-\infty}$ after co-administration of ketoconazole were 37 and $102 \%$, respectively, and the $90 \%$ CIs for the estimate of the ratio of least squares geometric means of $C_{\max }$ and $\mathrm{AUC}_{0-\infty}$ with and without ketoconazole exceeded $125 \%\left(C_{\max }, 123-153 \%\right.$; $\mathrm{AUC}_{0-\infty}, 177-230 \%$ ) (Table 3). Ketoconazole co-administration increased the $t_{\max }$ of R406 from $1.38 \mathrm{~h}$ (fostamatinib alone) to $1.69 \mathrm{~h}$ (fostamatinib plus ketoconazole) and the average $t_{1 / 2}$ increased from 14.2 to $18.4 \mathrm{~h}$ in association with ketoconazole co-administration.

\subsubsection{Verapamil Interaction Study}

Higher plasma levels of R406 were observed after co-administration of fostamatinib $(150 \mathrm{mg})$ and verapamil (80 $\mathrm{mg}$ three times daily) than after administration of fostamatinib alone (Figs. 2b, 3b). R406 AUC was higher after verapamil co-administration in 10 of the 13 subjects, with an average increase in the co-administration period of $39 \%$ (90\% CI for ratio, 108-180) (Table 3). There was no consistent effect of verapamil co-administration on R406 $C_{\max }$, least square geometric mean ratios for individual subjects ranging from 34 to $275 \%$ with an average of $106 \%$ and a $90 \%$ CI of 78-144. Similarly, there was no consistent effect of verapamil on R406 $t_{\max }$. There did not appear to be any specific relationship between changes in $C_{\max }$ and $t_{\max }$ (e.g., higher $C_{\max }$ was not always associated with later $\left.t_{\max }\right)$. The median $t_{\max }$ was similar between fostamatinib alone $(1.48 \mathrm{~h})$ and fostamatinib plus verapamil $(1.50 \mathrm{~h})$. Average $t_{1 / 2}$ increased by $41 \%$ from 13.5 to $19.1 \mathrm{~h}$ in association with verapamil co-administration (Table 3 ).

\subsubsection{Rifampicin Interaction Study}

Plasma levels of R406 were lower after co-administration of fostamatinib $(150 \mathrm{mg})$ and rifampicin $(600 \mathrm{mg}$ once daily) than after administration of fostamatinib alone (Figs. 2c, 3c). The geometric least squares mean ratios for AUC and $\mathrm{C}_{\max }$ were 25 and $41 \%$, respectively, with $90 \%$ CIs that were below $80 \%$ for both parameters $\left(C_{\max }\right.$, 30-56 \%; AUC, 19-32 \%) (Table 3). There was no consistent effect of rifampicin co-administration on $t_{\max }$, but median $t_{\max }$ values were shorter after rifampicin/fostamatinib co-administration $(1 \mathrm{~h})$ than after fostamatinib monotherapy $(2 \mathrm{~h})$. The geometric mean R406 $t_{1 / 2}$ for R406 decreased from 15.0 to $10.8 \mathrm{~h}$ in association with rifampicin co-administration (Table 3).

\subsection{Safety Assessments}

Fostamatinib was well tolerated in all three clinical studies, both when administered alone and in combination with a CYP3A4 inducer/inhibitor. There were no deaths, serious AEs, withdrawals due to AEs or severe AEs in any of the studies, nor were there any clinically relevant trends in laboratory test results, vital signs, physical findings, or other observations related to safety. 

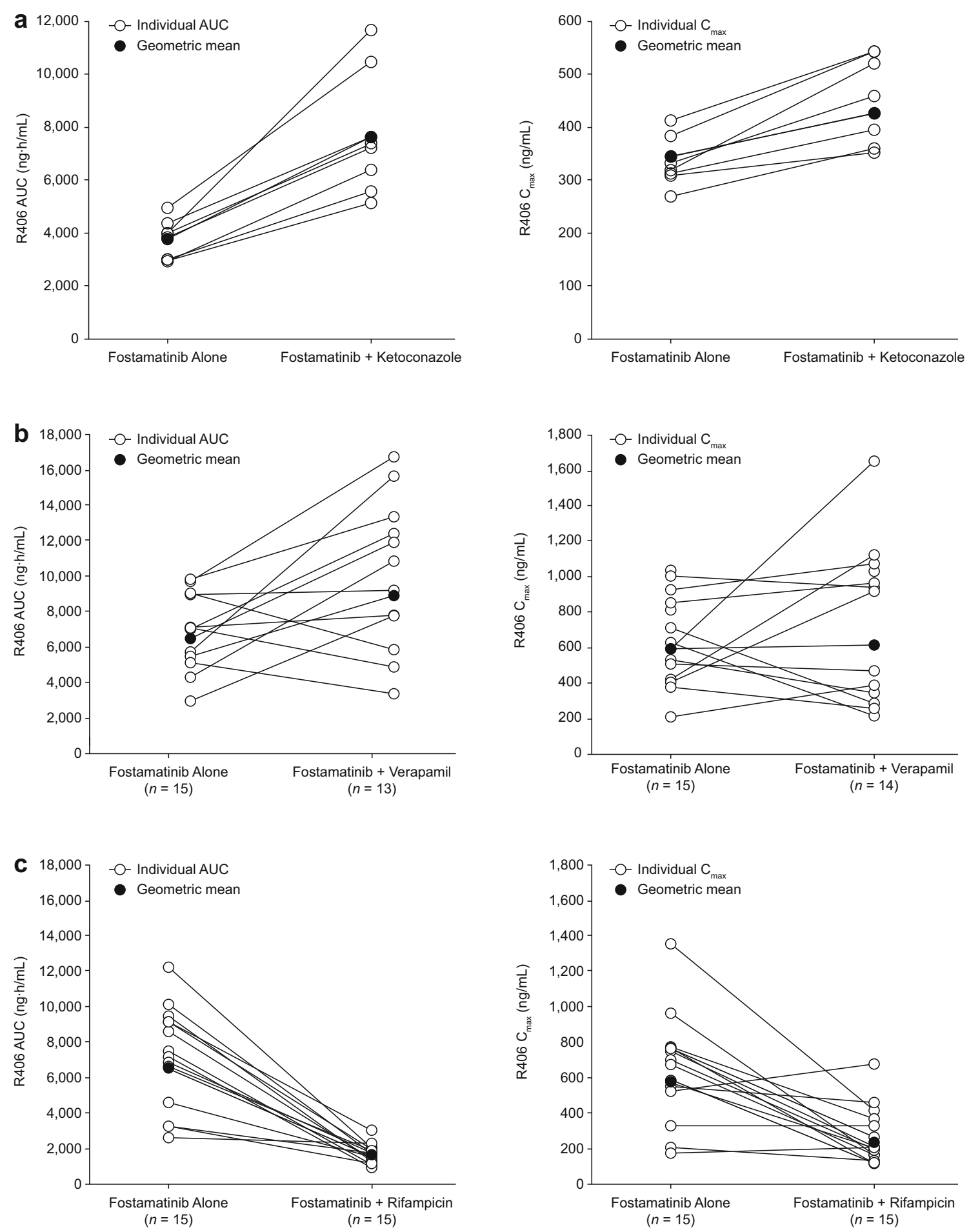

Fig. 3 Individual and geometric mean R406 $C_{\max }$ and AUC values in both treatment periods in the three clinical studies. a Ketoconazole interaction study. b Verapamil interaction study. c Rifampicin interaction study

\subsubsection{Ketoconazole Interaction Study}

A total of eight AEs, six of which were treatment-emergent, were reported. The two AEs that occurred prior to administration of fostamatinib comprised mild low back pain (after administration of ketoconazole) and mild headache (after placebo). The treatment-emergent AEs (headache, three events; low back pain, three events) were reported by five of the eight subjects, all of whom were receiving fostamatinib and placebo, placebo, or 


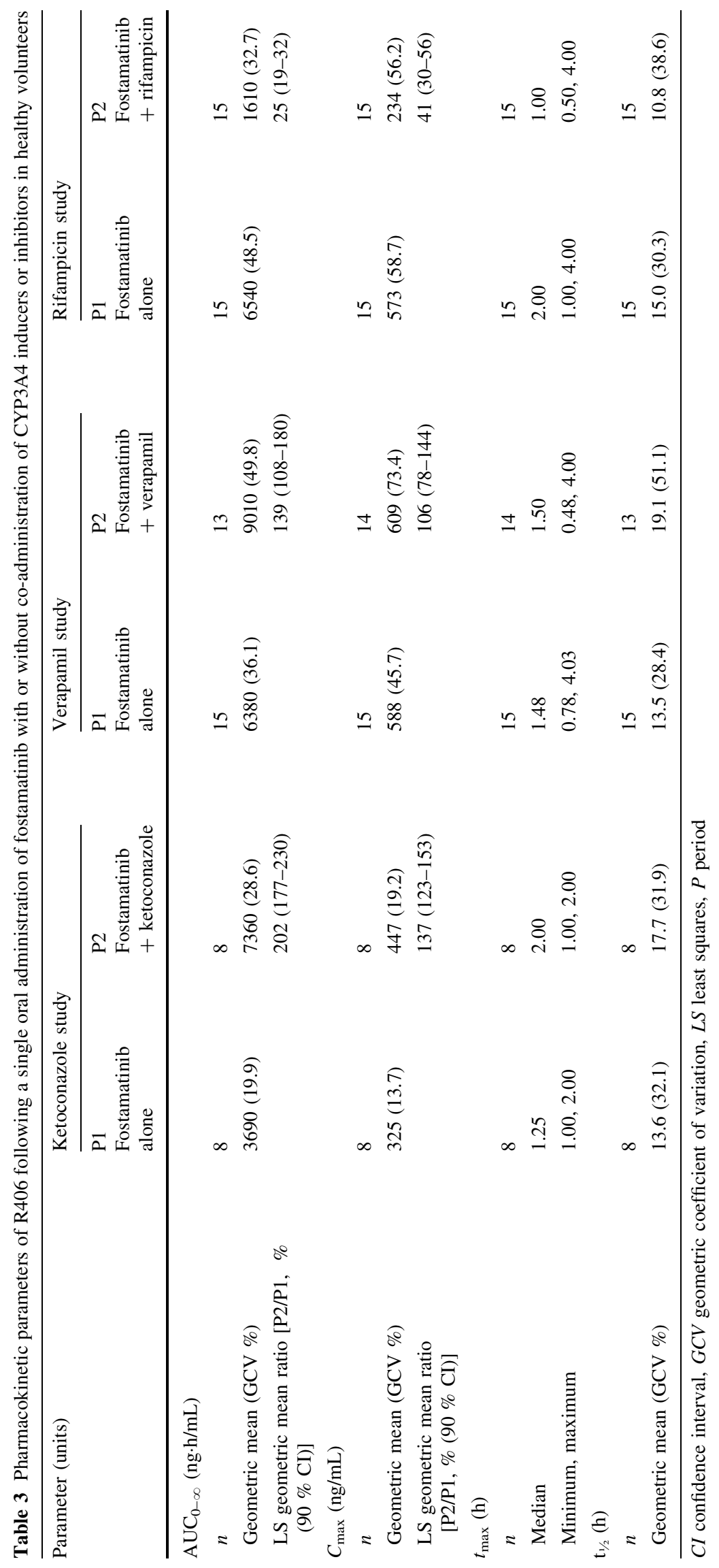


ketoconazole alone. No treatment-emergent AEs were reported by subjects receiving fostamatinib and ketoconazole. All treatment-emergent AEs were considered mild in intensity.

\subsubsection{Verapamil Interaction Study}

A total of three AEs in three patients (one mild, two moderate in severity) were reported. These occurred in all treatment arms (i.e., one $\mathrm{AE}$ per treatment arm reported). These comprised bacterial dermatitis, superficial phlebitis, and headache. None of these AEs were considered by the investigators to be related to administration of fostamatinib.

\subsubsection{Rifampicin Interaction Study}

A total of four AEs were recorded in this study, one in each of four subjects (one occurred in the rifampicin-only arm, two in the rifampicin/fostamatinib arm, and one in the fostamatinib-only arm). All were mild in intensity. One AE of pollakiuria (frequent urination), which began during rifampicin monotherapy, was regarded by the investigator as being causally related to rifampicin administration. The remaining AEs, all of which were considered unrelated to fostamatinib administration, comprised pain in an extremity (two subjects) and headache (one subject).

\section{Discussion}

Treatment of patients with RA is often complicated by the presence of comorbidities [6-8]. As a consequence, patients with RA often require polypharmacy. Drug-drug interactions (DDIs) are therefore a concern, and should be assessed. As CYP3A4 is involved in the metabolism of a substantial percentage (30-50\%) of drugs [11] (including R406, the active metabolite of fostamatinib), it is important to establish whether concomitant administration of CYP3A4 inducers or inhibitors affects the pharmacokinetics of fostamatinib or R406.

The in vitro work reported in this paper has demonstrated that R406 can be metabolized by an NADPH-dependent process, that CYP3A4 plays a major role in the oxidative metabolism of $\mathrm{R} 406$, and that the primary route of hepatic microsomal metabolism of R406 is para-Odemethylation. These findings concur with results obtained by Sweeny et al., who demonstrated that R406 undergoes both direct glucuronidation and CYP3A4-mediated para-Odemethylation [5]. The product of O-demethylation-the major metabolite of R406-is designated R529 [5]. R529 is further metabolized to a 3,5-benzene diol metabolite, which is the major fecal metabolite of fostamatinib in humans [5]. It is therefore evident that CYP3A4 plays an important role in the metabolism of R406. The only other isoform that was found to metabolize R406 in the current study was CYP2C9. However, hepatic microsomal levels of CYP3A4 are on average 5-fold higher than those of CYP2C9 (101.5 pmol/mg vs $18 \mathrm{pmol} / \mathrm{mg})[12,13]$. It can therefore be estimated that CYP3A4 accounts for $>96 \%$ of total CYP450 oxidative metabolism of R406 in human liver.

This in vitro work is supported by the results of the three clinical studies that explored the clinical relevance of CYP3A4 activity in fostamatinib-treated subjects, using agents that are typically used to determine the effects of CYP3A4 modulation on drug pharmacokinetics [10]. The studies reported herein indicate that ketoconazole, a strong CYP3A4 inhibitor, is associated with a 2-fold increase in exposure to R406 after administration of fostamatinib. It is likely that this ketoconazole-induced increase in R406 exposure is mediated via inhibition of CYP3A4 metabolism. However, based on the definitions in the current US Food and Drug Administration (FDA) guidance [14], R406 would not be considered a "sensitive substrate" of CYP3A. This designation is reserved for substrates that show $\geq 5$ fold increase in exposure in response to co-administration with strong CYP3A inhibitors [14]. Since completion of this study, the FDA has recommended that clarithromycin or itraconazole be used as an alternative strong CYP3A in drug interaction studies, rather than ketoconazole, to reduce the risk of liver injury [15].

The effect on R406 pharmacokinetics by verapamil, a moderate CYP3A4 inhibitor, was less marked than that of ketoconazole. However, there were still increases in AUC and $t_{1 / 2}$ of approximately $50 \%$, although no consistent effect on $C_{\max }$ and $t_{\max }$ was observed with verapamil coadministration. Co-administration of the CYP3A4 inducer, rifampicin, was associated with reductions of approximately $75 \%$ in R406 AUC and $60 \%$ in R406 $C_{\text {max }}$. This reduction in $\mathrm{R} 406$ exposure was associated with a decrease in $t_{1 / 2}$ and a shortening of median $t_{\max }$. Previous work has shown that rifampicin acts as an inducer of uridine diphosphate glucuronosyltransferase 1A9-mediated glucuronidation [16], and it is therefore possible that glucuronidation of R406 was also increased by co-administration of rifampicin. It is possible that ketoconazole, verapamil and rifampicin altered expression or activity of protein transporters including P-glycoprotein that may have contributed to the interactions observed.

These studies have demonstrated that pharmacokinetic interactions occur when fostamatinib is administered in combination with ketoconazole, verapamil, and rifampicin. It is possible that the decrease in fostamatinib exposure associated with rifampicin co-administration may lead to a decrease in efficacy. This should be taken into account 
when considering co-prescription of fostamatinib with such agents. It is a recognized limitation of the clinical studies performed that they are single dose studies that provide indicative pharmacokinetic DDI potential but may not fully characterize the DDI risk or safety in the patient population. As ketoconazole has been withdrawn in some territories, it may not be considered as an ideal potent CYP3A4 inhibitor probe but we believe the data do provide useful information to assess DDI risk through this mechanism.

No new safety concerns were documented during these three clinical studies. It is acknowledged, however, that these studies all involved administration of a single dose of fostamatinib to a small number of subjects - a design that is typical of DDI studies, but which is unable to explore the effects of long-term doubling of R406 exposure in humans.

It is unlikely that the difference among studies in fostamatinib formulation (ketoconazole study: suspension in orange juice; verapamil and rifampicin studies: tablets) affected the bioavailability of fostamatinib. Moreover, as no direct comparisons were made among the three studies, any difference in bioavailability would not affect the conclusions of each individual study.

\section{Conclusions}

The in vitro work presented herein has confirmed that the primary route of hepatic microsomal metabolism for R406 is O-demethylation and that oxidative metabolism of R406 is predominantly catalyzed by CYP3A4. The studies presented in this paper demonstrate that metabolism of, and exposure to, R406 (the active metabolite of fostamatinib) is affected by concomitant administration of the stated CYP3A4 inhibitors and inducers. Ketoconazole and verapamil increased exposure to R406 after administration of fostamatinib, probably via inhibition of CYP3A4-mediated metabolism. In contrast, R406 exposure was decreased after co-administration of the CYP3A4 inducer, rifampicin. It is likely that this was caused by induction of CYP3A4mediated metabolism. Overall, fostamatinib was well tolerated when administered alone or in combination with CYP3A4 inhibitors and inducers. These findings should be taken into account when considering co-prescription of fostamatinib with such agents.

Acknowledgments This study was sponsored by AstraZeneca. We acknowledge the editorial services of Shelley Lindley, PhD, and Safeer Mughal, PhD, from PAREXEL, which were funded by AstraZeneca.

\section{Compliance with Ethical Standards}

Disclosures Paul Martin, Mike Gillen, and Stuart Oliver are fulltime employees of AstraZeneca and hold stocks/shares in
AstraZeneca. Clive Brealey and David Millson are ex-full-time employees of AstraZeneca and hold stocks/shares in AstraZeneca. Elliott B. Grossbard, Muhammad Baluom, David Lau, and David Sweeny are employees of Rigel Pharmaceuticals, Inc. Tim Mant and Kelli Craven are employees of Quintiles. Tim Mant is also supported by the National Institute for Health Research (NIHR) Biomedical Research Centre at Guy's and St Thomas' NHS Foundation Trust and King's College London.

Ethical standards All clinical investigations were performed in accordance with the Declaration of Helsinki and the International Conference on Harmonization/Good Clinical Practice standards, and were approved by appropriate institutional review boards. Written informed consent was obtained from all subjects prior to initiation of the studies.

Open Access This article is distributed under the terms of the Creative Commons Attribution-NonCommercial 4.0 International License (http://creativecommons.org/licenses/by-nc/4.0/), which permits any noncommercial use, distribution, and reproduction in any medium, provided you give appropriate credit to the original author(s) and the source, provide a link to the Creative Commons license, and indicate if changes were made.

\section{References}

1. Baluom M, Samara E, Grossbard EB, et al. Fostamatinib, a Sykkinase inhibitor, does not affect methotrexate pharmacokinetics in patients with rheumatoid arthritis. J Clin Pharmacol. 2011;51:1310-8.

2. Dawes P, Dimic A, Genovese M, et al. OSKIRA-2: a phase III, multicenter, randomized, double-blind, placebo-controlled parallel-group study of 2 dosing regimens of fostamatinib in rheumatoid arthritis patients with an inadequate response to disease-modifying antirheumatic drugs (Abstr. 455). Arthritis Rheum. 2013;65(10 Suppl):S198.

3. Genovese M, van der Heijde D, Keystone E, et al. OSKIRA-3: a phase III, multicenter, randomized, double-blind, placebo-controlled parallel-group study of 2 dosing regimens of fostamatinib in rheumatoid arthritis patients with an inadequate response to a tumor necrosis factor- $\alpha$ antagonist (Abstr. 456). Arthritis Rheum. 2013;65(10 Suppl):S199.

4. Weinblatt M, Genevese MC, Ho M, et al. OSKIRA-1: a phase III, multicenter, randomized, double-blind, placebo-controlled parallel-group study of 2 dosing regimens of fostamatinib in rheumatoid arthritis patients with an inadequate response to methotrexate (Abstr. 1793). Arthritis Rheum. 2013;65(10 Suppl):S763.

5. Sweeny DJ, Li W, Clough J, et al. Metabolism of fostamatinib, the oral methylene phosphate prodrug of the spleen tyrosine kinase inhibitor R406 in humans: contribution of hepatic and gut bacterial processes to the overall biotransformation. Drug Metab Dispos. 2010;38:1166-76.

6. Briggs AM, March L, Lassere M, et al. Baseline comorbidities in a population-based cohort of rheumatoid arthritis patients receiving biological therapy: data from the Australian rheumatology association database. Int J Rheumatol. 2009;2009:861481.

7. Turesson C, Jacobsson LT, Matteson EL. Cardiovascular comorbidity in rheumatic diseases. Vasc Health Risk Manag. 2008;4:605-14.

8. Nurmohamed MT. Cardiovascular risk in rheumatoid arthritis. Autoimmun Rev. 2009;8:663-7. 
9. Treharne GJ, Douglas KM, Iwaszko J, et al. Polypharmacy among people with rheumatoid arthritis: the role of age, disease duration and comorbidity. Musculoskelet Care. 2007;5:175-90.

10. Zhou SF. Drugs behave as substrates, inhibitors and inducers of human cytochrome P450 3A4. Curr Drug Metab. 2008;9:310-22.

11. Haddad A, Davis M, Lagman R. The pharmacological importance of cytochrome CYP3A4 in the palliation of symptoms: review and recommendations for avoiding adverse drug interactions. Support Care Cancer. 2007;15:251-7.

12. Nakajima $M$, Nakamura $S$, Tokudome $S$, et al. Azelastine N-demethylation by cytochrome P-450 (CYP)3A4, CYP2D6, and CYP1A2 in human liver microsomes: evaluation of approach to predict the contribution of multiple CYPs. Drug Metab Dispos. 1999;27:1381-91.

13. Koukouritaki SB, Manro JR, Marsh SA, et al. Developmental expression of human hepatic CYP2C9 and CYP2C19. J Pharmacol Exp Ther. 2004;308:965-74.
14. Center for Drug Evaluation and Research (CDER). Guidance for industry: drug interaction studies-study design, data analysis, implications for dosing, and labeling recommendations. US Food and Drug Administration Website. http://www.fda.gov/Drugs/ GuidanceComplianceRegulatoryInformation/Guidances/default. htm. Accessed 2 Sept 2013.

15. US Department of Health and Human Services, Food and Drug Administration. FDA advises against using oral ketoconazole in drug interaction studies due to serious potential side effects. US Food and Drug Administration Website. http://www.fda.gov/ Drugs/DrugSafety/ucm371017.htm. Accessed 16 Jan 2014.

16. Soars MG, Petullo DM, Eckstein JA, et al. An assessment of udpglucuronosyltransferase induction using primary human hepatocytes. Drug Metab Dispos. 2004;32:140-8. 